\title{
Decompressive hemicraniectomy for acute ischemic stroke in a patient implanted with a left ventricular assist device: a case report
}

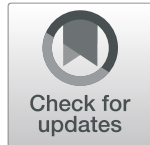

Walid Oulehri ${ }^{1,2^{*}}$ (D), Mircea Cristinar ${ }^{1}$, Gharib Ajob ${ }^{1}$, Sandrine Marguerite ${ }^{1}$, Bob Heger ${ }^{1,2}$, Hélène Cebula ${ }^{3}$, Michel Kindo ${ }^{4,2}$ and Paul Michel Mertes ${ }^{1,2}$

\begin{abstract}
Background: Thromboembolic ischemic stroke (IS) is one of the most feared complications of left ventricular assist device (LVAD) placement and represents a challenge to surgical management because of concomitant anticoagulant therapy.

Case presentation: A 39-year-old man presented with cardiogenic shock following an out-of-hospital cardiac arrest. After a period of stabilization, the patient was referred for LVAD placement. Upon recovery from anesthesia, he presented with acute neurological deficits suggestive of IS. A brain computed tomography confirmed the diagnosis, and an emergency decompressive hemicraniectomy (DHC) was performed. Anticoagulation was managed empirically. The patient's neurological status progressively improved and he was referred for heart transplantation at five months from DHC. One month later, cranioplasty was performed.

Conclusions: This report suggests an anticoagulation management approach in combination with decompressive craniectomy after IS in a patient with LVAD placement was successful. An optimized anticoagulation management and collaborative team-based practice may contribute to successful outcomes in complex cases.
\end{abstract}

Keywords: Decompressive craniectomy, Cardiac arrest, Left ventricular assist device, Ischemic stroke, Anticoagulation, Heart transplantation

\section{Background}

Heart failure (HF) is a major public health concern resulting in substantial morbidity, mortality, and healthcare expenditures worldwide. Heart transplantation (HT) remains the treatment of choice for improving survival in patients suffering from advanced HF, although organ shortage restricts this option. In this scenario, a left ventricular assist device (LVAD) can be implanted to

\footnotetext{
* Correspondence: walid.oulehri@chru-strasbourg.fr

'Pôle Anesthésie Réanimation Chirurgicale, service de Réanimation

Chirurgicale, Hôpitaux Universitaires de Strasbourg NHC, 1, Place de I'Hôpital, 67000 Strasbourg, France

EA 3072, Fédération de Médecine Translationnelle de Strasbourg, Institut de Physiologie, Université de Strasbourg, 67000 Strasbourg, France

Full list of author information is available at the end of the article
}

bridge patients to HT. The optimal anticoagulation strategy to avoid thrombotic complications (e.g., pump thrombosis and stroke) in patients with LVADs remains an ongoing challenge especially in complex clinical situations. To our knowledge, there is still no consensus on how to best manage patients with LVADs who require emergency decompressive hemicraniectomy (DHC) following a malignant ischemic stroke (IS). In the present case report, we describe how an optimized anticoagulation management and collaborative team-based practice are paramount to prevent catastrophic outcomes in such challenging cases.

(c) The Author(s). 2020 Open Access This article is licensed under a Creative Commons Attribution 4.0 International License, which permits use, sharing, adaptation, distribution and reproduction in any medium or format, as long as you give appropriate credit to the original author(s) and the source, provide a link to the Creative Commons licence, and indicate if changes were made. The images or other third party material in this article are included in the article's Creative Commons licence, unless indicated otherwise in a credit line to the material. If material is not included in the article's Creative Commons licence and your intended use is not permitted by statutory regulation or exceeds the permitted use, you will need to obtain permission directly from the copyright holder. To view a copy of this licence, visit http://creativecommons.org/licenses/by/4.0/. The Creative Commons Public Domain Dedication waiver (http://creativecommons.org/publicdomain/zero/1.0/) applies to the data made available in this article, unless otherwise stated in a credit line to the data. 


\section{Case presentation}

A 39-year-old man - with a negative personal history for cardiovascular disorders and previous surgery - presented with cardiogenic shock following an out-ofhospital cardiac arrest. Resuscitation and emergency coronary angioplasty were successfully implemented, followed by admission to the intensive care unit. Antiplatelet therapy was started with aspirin $75 \mathrm{mg}$ per day. Because of tachyarrhythmia-related cardiovascular compromise, the patient was placed on veno-arterial extracorporeal life support (VA-ECLS). However, the acute onset of pulmonary edema required placement of a LAVD (Impella ${ }^{\circ}$ 5.0; Abiomed Inc.; Danvers, MA, USA). A brain computed tomography $(\mathrm{CT})$ scan following leftsided seizure onset revealed a right cerebellar infarct. After approximately two weeks, Impella ${ }^{\circ} 5.0$ was removed and successfully replaced by a centrifugal continuous-flow ventricular assist device (HVAD $^{\circ}$; HeartWare ${ }^{\circ}$ Medtronic, Minneapolis, MN, USA). Aspirin dosing was not tapered off because of the previous coronary angioplasty. Seven hours after recovery from anesthesia, acute neurological deficits suggestive of IS (left hemiparesis; Glasgow Coma Scale score: 10) were evident. The patient was intubated and verbal responses were not assessable. After consultation with neurologists, endovascular treatment was deemed unfeasible because of delayed discovery of the neurological presentation. A $\mathrm{CT}$ scan performed at $20 \mathrm{~h}$ post-admission revealed a complete right sylvian IS with significant mass effect resulting in a midline shift of $4.8 \mathrm{~mm}$ in the absence of haemorrhagic transformation (Fig. 1). In light of the rapidly deteriorating neurological conditions and imaging evidence of IS, emergency DHC was performed after weighing surgical-related risks against potential benefits (Figs. 2 and 3). Aspirin $75 \mathrm{mg} /$ day was stopped the day before surgery and no intraoperative prothrombotic medication was given. The pre-operative laboratory coagulation status was as follows: international normalized ratio (INR), 1.34; activated partial thromboplastin time (aPTT) ratio, 1.2; platelet count: $367,000 / \mu \mathrm{L}$. Platelet function tests were not performed albeit expected to be impaired. Previous antiplatelet therapy did not technically affect emergency DHC. Anticoagulation (continuous infusion of unfractionated heparin at a dose of 25,000 $\mathrm{UI} / \mathrm{mL}$ ) was started by consensus of neurosurgeons and cardiac surgeons on the second post-DHC day. Unfractionated heparin infusion was titrated to achieve a target aPTT ratio of 1.2 for the first week and 1.8-2 for two subsequent weeks. Antiplatelet treatment was reintroduced two weeks after emergency DHC. Finally, unfractionated heparin infusion was titrated up to obtain an optimal aPTT ratio of $2.5-3$. On the third post-DHC week, the patient was switched to oral anticoagulation. During the first two post-DHC weeks, the patient received empirical therapy with levetiracetam to prevent potential seizures elicited by brain edema.

Upon recovery from anesthesia after DHC, the patient was conscious and responsive to commands. Although left-side paralysis initially persisted, there was a gradual recovery in neurological symptoms that allowed transfer to a multidisciplinary rehabilitation center. Cardiac flows remained stable under control of the $\mathrm{HVAD}^{\circ}$ device. The patient was referred for $\mathrm{HT}$ at five months from DHC, followed by cranioplasty one month thereafter. Both intra- and postoperative courses were uneventful.

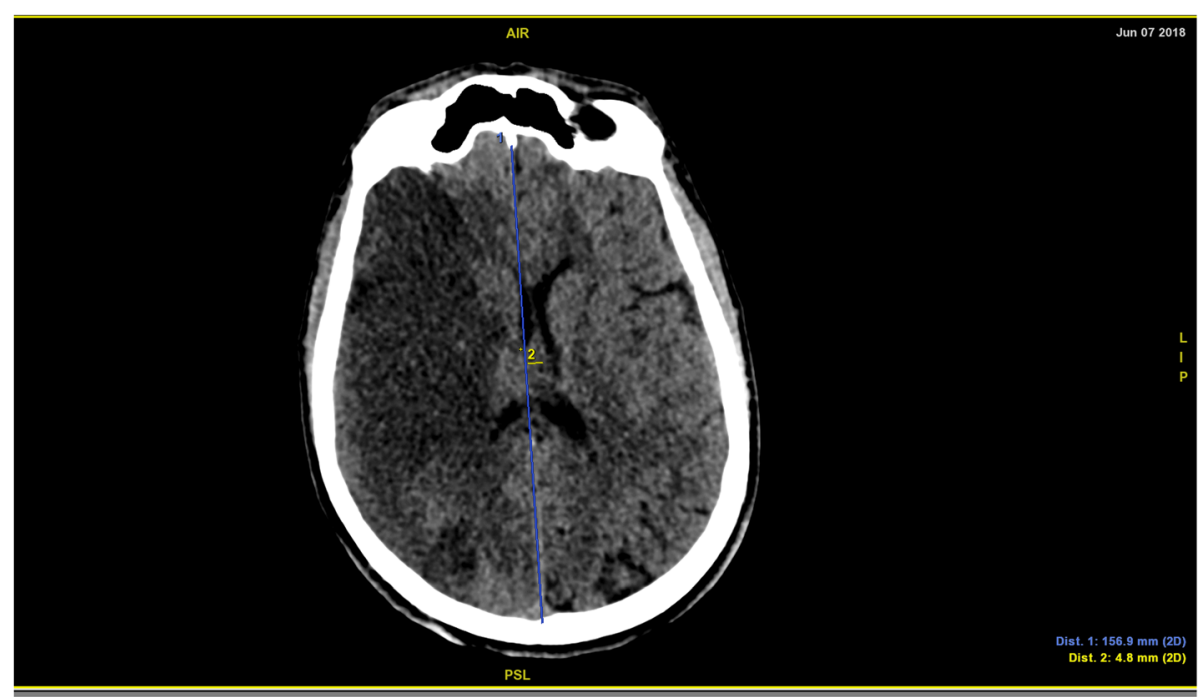

Fig. $1 \mathrm{~A}$ brain computed tomography scan without contrast revealed a right sylvian ischemic stroke with a mass effect and a midline shift of $4.8 \mathrm{~mm}$ 


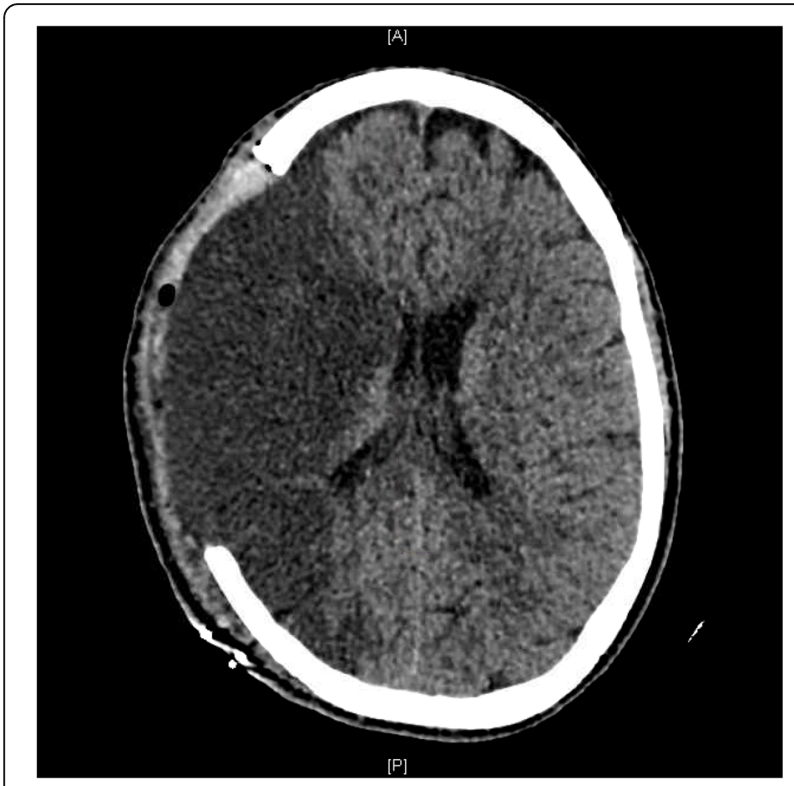

Fig. 2 A brain computed tomography scan illustrating right decompressive hemicraniectomy

\section{Discussion and conclusions}

We describe a case of malignant IS following the implantation of a $\mathrm{HVAD}^{\circ}$ device who was successfully treated with emergency DHC. Cestari et al. [1] have previously shown the clinical utility of LVADs for improving survival of patients with advanced HF. The reported prevalence of IS following LVAD implantation vary widely from 2 to $14 \%$ [2]. Unfortunately, early identification of neurological deterioration may be challenging owing to the prolonged post-operative clinical course. Although brain edema in the context of a LVAD-related

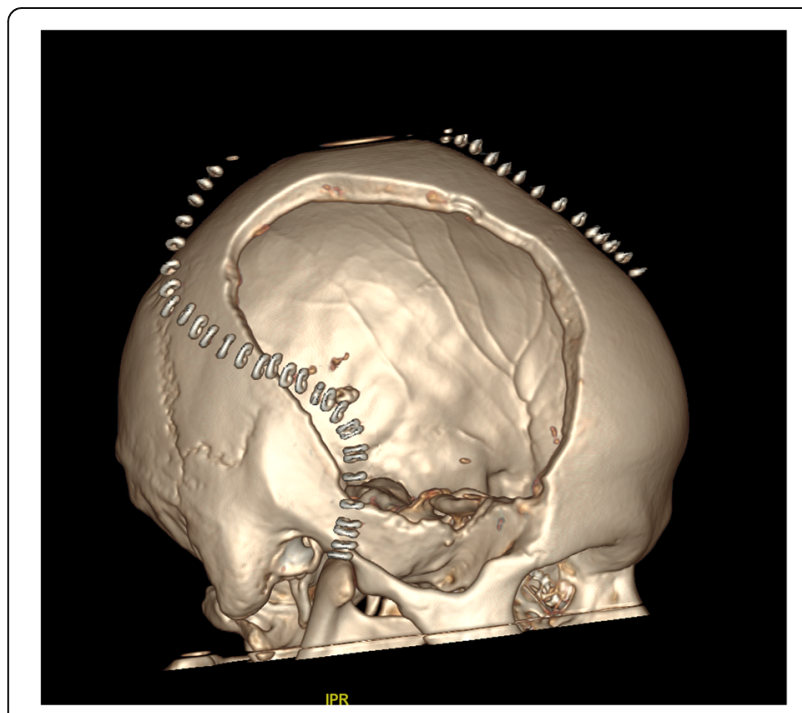

Fig. 3 Three-dimensional rendering of the right decompressive hemicraniectomy stroke generally portends a poor prognosis [3], emergency DHC in our patient was successful and allowed regaining an acceptable neurological function before HT. Both size and location of DHC affect the treatment effect. Even if DHC was quite small it allowed for the patient good functional results. A previous study in the general population demonstrated that implementation of DHC within the first $24 \mathrm{~h}$ of an IS can lead to successful outcomes in young patients without low preoperative Glasgow coma scale scores [4]. However, the question as to whether these findings are applicable to patients implanted with a LVAD has not been previously addressed.

Patients with a history of LVAD implantation who develop an IS may be continued on anticoagulation and antiplatelet treatment. In our case, decisions on anticoagulation management were taken by consensus within an interdisciplinary team of cardiac surgeons, neurosurgeons, neurovascular physicians, and anesthesiologists. Our patient underwent emergency DHC following a temporary two-day interruption of previous anticoagulation therapy. Low-dose anticoagulation with continuous infusion of unfractionated heparin was started in the post-DHC phase, followed by the reintroduction of antiplatelet therapy. We are unaware of guidelines or standard protocols for implementing anticoagulation or antiplatelet therapy in patients implanted with a LVAD who had undergone neurosurgery. In this scenario, pump thrombosis should be aggressively prevented [5] through a careful monitoring of pump speed while preserving aortic ejection and maintaining an optimal blood pressure control. LVAD failure did not occur in our case and - possibly because of his young age coupled with optimized anticoagulation management - the patient's neurological status progressively improved, being finally referred for HT at five months after DHC.

In light of our patient's successful clinical course, DHC seems to be possible in young patients who develop IS after LVAD placement. An optimized anticoagulation management and collaborative team-based practice are paramount to ensure positive outcomes in such highly complex cases. Despite these good results this type of handling might merit further investigation in a higher study setting.

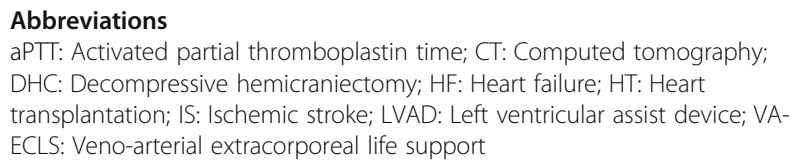

\section{Acknowledgements}

None.

\section{Authors' contributions}

WO was the main investigator, analyzed the relevant clinical records, and drafted the manuscript. MC, GA, SM, BH, and $\mathrm{HC}$ collected clinical records and contributed to the patient's management. MK and PMM revised the 
manuscript for important intellectual content. All authors have read and approved the manuscript.

\section{Funding}

Not applicable.

\section{Availability of data and materials}

Not applicable. All data supporting the conclusions are presented in the manuscript.

\section{Ethics approval and consent to participate}

Written informed consent was obtained from the patient to use this case and any accompanying images for the purpose of journal manuscript publication. A copy of the written consent is available for review by the journal. Ethical approval was not necessary as this is a case study.

\section{Consent for publication}

Written consent for publication was obtained from the patient in this case study.

\section{Competing interests}

The authors declare that they have no competing interests.

\section{Author details}

'Pôle Anesthésie Réanimation Chirurgicale, service de Réanimation Chirurgicale, Hôpitaux Universitaires de Strasbourg NHC, 1, Place de l'Hôpital, 67000 Strasbourg, France. ${ }^{2}$ EA 3072, Fédération de Médecine Translationnelle de Strasbourg, Institut de Physiologie, Université de Strasbourg, 67000 Strasbourg, France. ${ }^{3}$ Pôle Tête et Cou, service de Neurochirurgie, Hôpitaux Universitaires de Strasbourg NHC, 67000 Strasbourg, France. ${ }^{4}$ Pôle Cardiologie, service de Chirurgie Cardiovasculaire, Hôpitaux Universitaires de Strasbourg NHC, 67000 Strasbourg, France.

Received: 16 August 2019 Accepted: 4 June 2020

Published online: 10 June 2020

\section{References}

1. Cestari V, Pessoa VL, de Souza Neto JD, Moreira T, Florêncio R, de Vasconcelos GG, et al. Clinical evolution of patients using ventricular assist devices as a bridge for transplantation. Transplant Proc. 2018;50:796-803.

2. Frontera JA, Starling R, Cho S-M, Nowacki AS, Uchino K, Hussain MS, et al. Risk factors, mortality, and timing of ischemic and hemorrhagic stroke with left ventricular assist devices. J Heart Lung Transplant. 2017;36:673-83.

3. Bishawi M, Joseph J, Patel C, Schroder J, Daneshmand M, Bowles D, et al. Risk factors for stroke on left ventricular assist devices. J Card Surg. 2018;33: 348-52.

4. Raffiq MA, Haspani MS, Kandasamy R, Abdullah J. Decompressive craniectomy for malignant middle cerebral artery infarction: impact on mortality and functional outcome. Surg Neurol Int. 2014;5:102.

5. Maltais S, Kilic A, Nathan S, Keebler M, Emani S, Ransom J, et al. PREVENtion of HeartMate II pump thrombosis through clinical management: the PREVENT multi-center study. J Heart Lung Transplant. 2017;36:1-12.

\section{Publisher's Note}

Springer Nature remains neutral with regard to jurisdictional claims in published maps and institutional affiliations. 